\title{
Recht aus der Sicht der evolutorischen Ökonomik
}

\author{
Citation for published version (APA):
}

Backhaus, J. G. (2000). Recht aus der Sicht der evolutorischen Ökonomik. METEOR, Maastricht University School of Business and Economics. METEOR Research Memorandum No. 001 https://doi.org/10.26481/umamet.2000001

Document status and date:

Published: 01/01/2000

DOI:

10.26481/umamet.2000001

Document Version:

Publisher's PDF, also known as Version of record

\section{Please check the document version of this publication:}

- A submitted manuscript is the version of the article upon submission and before peer-review. There can be important differences between the submitted version and the official published version of record.

People interested in the research are advised to contact the author for the final version of the publication, or visit the DOI to the publisher's website.

- The final author version and the galley proof are versions of the publication after peer review.

- The final published version features the final layout of the paper including the volume, issue and page numbers.

Link to publication

\footnotetext{
General rights rights.

- You may freely distribute the URL identifying the publication in the public portal. please follow below link for the End User Agreement:

www.umlib.nl/taverne-license

Take down policy

If you believe that this document breaches copyright please contact us at:

repository@maastrichtuniversity.nl

providing details and we will investigate your claim.
}

Copyright and moral rights for the publications made accessible in the public portal are retained by the authors and/or other copyright owners and it is a condition of accessing publications that users recognise and abide by the legal requirements associated with these

- Users may download and print one copy of any publication from the public portal for the purpose of private study or research.

- You may not further distribute the material or use it for any profit-making activity or commercial gain

If the publication is distributed under the terms of Article $25 \mathrm{fa}$ of the Dutch Copyright Act, indicated by the "Taverne" license above, 


\title{
Recht aus der Sicht der evolutorischen Ökonomik
}

\author{
Prof. Dr. Jürgen G. Backhaus \\ Department of Economics \\ Maastricht University \\ P.O. Box 616 \\ 6200 MD Maastricht \\ The Netherlands \\ tel: $+31-43-3883636$ \\ fax: $+31-43-3884878$ \\ email: j.backhaus@algec.unimaas.nl
}

Beitrag für das Handbuch der evolutorischen Ökonomik

Carsten Herrmann-Pillath und Marco Lehmann-Waffenschmidt (Hrsg.)

Heidelberg: Springer-Verlag

\begin{abstract}
$\mathrm{T}$

This entry for a Handbook of Evolutionary Economics offers a short sketch of the applicability of evolutionary economics to law, and in particular to the economic analysis of law. The entry has three main sections, emphasizing in turn issues of transaction costs, the concept of the legal system in economic analyses and different scenarios of legal change.
\end{abstract}

JEL codes: K00, O38

keywords: evolution of the law, system change, technological change 


\section{Recht aus der Sicht der evolutorischen Ökonomik}

\section{Einleitung}

"Evolution", so definiert Witt (1994, S. 83), "ist ein Prozeß während dessen sich ein System im Laufe der Zeit aus sich selbst heraus verändert und transformiert." ${ }^{\text {"l }}$ Unter evolutorischen Gesichtspunkten interessiert uns also das Recht in seinem Wandel, insbesondere dann, wenn dieser Wandel als in einem System endogen ablaufend dargestellt werden kann. Diese Definition hilft insoweit, den Stoff $\mathrm{zu}$ gliedern, als gewiß drei Definitionselemente berücksichtigt werden müssen. Zum einen ist ein System des Rechts zu definieren, das dem Wandel unterliegt. Ein System setzt systematisch auf einander bezogene Teile voraus, und es muß Deutlichkeit darüber herrschen, welche Teile (des Rechts) zum System gehören und welche nicht. Das Rechtssystem besteht notwendigerweise in einem Umfeld, das aus anderen Teilen des Rechts, aber auch anderen Normierungen, Sitten, Gebräuchen, usw. bestehen kann. Gewiß können diese auch wiederum ein System bilden.

Der zweite Aspekt betrifft den Wandel. Der Wandel kann einmal exogen das System erfassen oder endogen aus dem System selbst heraus erfolgen. Der evolutorische Aspekt betrifft die zweite Möglichkeit. Das Rechtssystem soll sich aus sich selbst heraus verändern und erneuern. Hier sind drei Aspekte bedeutsam. Der erste Aspekt betrifft den Anlaß des Wandels, der zweite den Motor des Wandels und der dritte die Frage, ob der Wandel oder die Transformation stabil ist in dem Sinne, daß sich das System tatsächlich erneuert und nicht degeneriert.

Damit ist drittens die Frage verknüpft, ob das System aus sich heraus zu einem evolutorischen Wandel in der Lage ist, oder ob der Wandel exogen erfolgen muß, kann oder wird.

Wenn ein System aus sich heraus nicht (mehr) zum Wandel fähig ist, dann befindet sich dieses System in einer Systemkrise. Es wird nötig sein, entweder die Ursache zu beseitigen, die den Wandel verhindert, oder aber das System neu zu konstituieren. Man muß insofern bei den Ursachen des Wandels beginnen. Erst danach läßt sich sinnvollerweise (in telelogischer Absicht) das System abgrenzen. Deshalb werden im Folgenden die Fragen 1 und 2 in der Reihenfolge umgekehrt.

Die folgenden Ausführungen bestehen neben diese Einleitung aus drei Teilen. Teil 1 ist den Ursachen des Wandels gewidmet, also einerseits dem Grund der Bewegung und andererseits dem Anlaß. Teil 2 handelt von der Abgrenzung des Rechtssystems, und Teil 3 von der Alternative zwischen exogenem und endogenem Wandel. Alle drei Aspekte werden mit

\footnotetext{
${ }^{1}$ Im englischen Original: "Evolution is a process in which a system under consideration transforms itself over time".
} 
Beispielen aus der Rechtsgeschichte illustriert. Im zusammenfassenden Ausblick geht es um einige wichtige Fragen, die in der nahen Zukunft mit Hilfe der evolutorischen Rechtsökonomie bearbeitet werden können.

\section{I.}

Im Zentrum eines evolutorischen Ansatzes zur Erklärung des Rechts, und damit des rechtlichen Wandels, müssen stets die Transaktionskosten stehen. Ganz allgemein sind dies die Kosten der Nutzung des Rechts. Da das Recht inhaltlich offen ist, also gute oder schlechte, mehr oder weniger durchdachte Handlungen, vor allem Austauschhandlungen mit seiner Hilfe möglich gemacht werden können, ist man bei der ökonomischen Rechtsanalyse auf die Kostenseite verwiesen. Gewiß ist die Nutzung einer rechtlichen Möglichkeit vom Nettoertrag abhängig, also der Differenz zwischen dem Bruttoertrag und der Nutzung des Rechts. Wenn wir (Coase, 1937) vor der Entscheidung stehen, ob eine bestimmte Fertigung innerhalb der Unternehmung oder außerhalb der Unternehmung stattfinden soll, dann müssen wir bei bekannter Technologie entscheiden, ob die Koordination innerhalb der Unternehmung leichter ist oder die Auslagerung in eine andere, die dann, da ein Rechtsverhältnis begründet werden muß, eine Abwägung der jeweiligen Kosten erforderlich macht. Bei bekannte Technologie und vorausgesetzt, daß wir uns über das Produkt einig sind, müssen wir nun nur noch abwägen, ob der Vorgang innerhalb oder außerhalb der Unternehmung günstiger, voraussehbarer, fehlerunanfälliger und insgesamt reibungsloser ablaufen kann. Man kann nun unterstellen, daß jenes Verfahren gewählt wird, dessen Nettoertrag erwartungsgemäß höher ist, daß also die Kosten der Nutzung entweder des unternehmensinternen Koordinationsverfahrens oder aber des Rechtssystems bei Auslagerung aus der Unternehmung so gering wie möglich gehalten werden sollen. In diesem Sinne sollen die Transaktionskosten minimiert werden. Diese Kosten können aber insbesondere bei komplexen Produktionsvorgängen immer sehr erheblich sein, sie können überhaupt den größten Teil der Produktionskosten ausmachen. Dies gilt insbesondere dann, wenn das Produkt diffus und schwer beschreibbar ist, zum Beispiel eine Beratung, ein Forschungsvorgang, eine künstlerische Produktion oder zum Beispiel ein riskanter medizinischer Eingriff. Das letzte Beispiel zeigt, daß die Transaktionskosten so hoch sein können, daß die Handlung überhaupt unterbleibt. ${ }^{2}$

Mann konnte sich fragen, ob Transaktionskosten auf allen Rechtsgebieten eine so zentrale Rolle spielen, daß sie überhaupt als der Motor des Wandels angesehen werden können. Dies ist durchaus der Fall. Im Verwaltungsrecht sind wir ständig mit neuen Ablaufformen konfrontiert, die noch vor Kurzem undenkbar gewesen wären. Dies gilt insbesondere für das leistende Verwaltungsrecht, etwa im Sozialrecht. Auch im Staatrecht finden wir eine

2 Dies gilt zum Beispiel dann, wenn sich der Arzt nicht freizeichnen kann und entweder keine Versicherungspolicen auf dem Markt erhältlich sind, oder aber die Versicherungsprämien die Differenz aus dem zu erwartendem Honorar und allen Kosten, insbesondere auch den Opportunitätskosten des Arztes übersteigen. 
erstaunliche Fülle von neuen Formen (Rechtsformen), Ablaufformen (Kontrollverfahren), die allesamt ihre Ursache darin haben, daß die klassische Formen und Verfahren zu aufwendig geworden sind, entweder weil der Beamtenapparat sie nicht mehr bewältigen kann, oder weil sie zu prozeßanfällig sind oder weil die Aufgabenfülle zu stark gewachsen ist. Ähnliches gilt im Familienrecht, und zwar sowohl im positiven als auch im dispositiven. Wir könnten die Liste weiterführen, über das Strafrecht, das Kirchenrecht, sogar bis hin ins Prozeßrecht. Überall treffen wir auf das Bemühen, dasselbe Ergebnis mit einem geringeren Aufwand an Transaktionskosten zu erreichen.

Transaktionskosten werden in der Regel in drei Fälle unterschieden, wobei die Einzelunterscheidung vom zu analysierenden Einzelfall abhängt und gegebenenfalls sehr viel tiefer gehen kann. Allgemein können wir unterscheiden die Kosten, die vor der Begründung eines Austauschverhältnisses entstehen, die Kosten, die mit der Begründung eines Austauschverhältnisses entstehen und die Kosten, die nach der Begründung eines Austauschverhältnisses entstehen. Das Austauschverhältnis selbst, der Vertrag (contract) muß verhandelt, begründet, vielleicht notariell bestätigt, formuliert, überprüft und unterschrieben werden. Etwa in diesem Rahmen bewegen sich die Kosten der Vertragsbegründung (contracting costs). Der einmal so geschlossene Vertrag muß aber auch eingehalten werden, und diese Einhaltung zu überprüfen und zu erzwingen ist je schwieriger, desto komplexer der Vertragsinhalt ist und desto diffuser die Umschreibung des Produktes, also der Vertragsleistung. In allen drei Phasen, aber insbesondere in der ersten Phase vor der Vertragsschließung spielt die Informationssuche eine besonders große Rolle. Korrekte Information ist sowohl beim Vertragsschluß (contracting) als auch bei der Vertragsüberwachung (policying) wichtig, aber vorab geht die Informationssuche (information search). Insofern können wir grob die drei Formen der Transaktionskosten unterscheiden, jene der Vertragsschließung (Contracting), jene der Vertragsüberwachung (Policying) und jene der Informationssuche (Information gathering). Insofern spricht man von den Transaktionskosten in der empirischen Literatur auch kurz von den CPI costs.

Da nun die Chance, für eine bestimmte wirtschaftliche Handlung das Recht auch tatsächlich zu nutzen, von der relativen Höhe dieser Transaktionskosten abhängt, steht jedes Rechtssystem ständig unter dem Druck, diese Transaktionskosten wo möglich zu senken. Die Suche nach niedrigeren Transaktionskosten nimmt, je nachdem welches Rechtssystem (siehe Teil 2) wir betrachten, ganz unterschiedliche Formen an. Innerhalb eine Organisation ist die Minimierung der Transaktionskosten trivial, da sich der Eigentïmer ja den daraus erzielten Ertrag unmittelbar aneignen kann. Wichtiger sind die Beziehungen außerhalb von Organisationen, also zwischen natürlichen Personen, zwischen Rechtspersonen, zwischen Rechtspersonen und natürlichen Personen usw. Im allgemeinen haben die Vertragspartner daran ein Interesse, die Transaktionskosten (relativ) so niedrig wie möglich zu halten, um den gemeinsamen Gewinn aus dem Vertragsverhältnis nicht unnötig $\mathrm{zu}$ schmälern. $\mathrm{Da}$ sie beide dem zu wählenden Vorgehen zustimmen müssen, kann sich nicht einer für ein System mit hohen Transaktionskosten entscheiden, die er etwa dem anderen überwälzen könnte. Beide 
Parteien haben ein Interesse daran, insofern interessenparallel, die Transaktionskosten zu senken. Natürlich handelt es sich hier nicht um den Versuch, die absoluten Transaktionskosten zu senken, sondern nur die relativen im Hinblick auf den Transaktionsgewinn, der erzielt werden soll. Wer auf den Fischmarkt geht, wird vor allem gegen Ende der Marktzeit mit dem Händler versuchen zu handeln (haggling), aber er läßt es darüber nicht zum Streit kommen. Auch der Händler gibt lieber klein bei, als auf dem verderblichen Fisch sitzenzubleiben. Jedenfalls bemüht keiner der beiden einen Notar. Geht es um ein kompliziertes Immobiliengeschäft eventuell noch mit Erbverträgen und Testamenten, ist man gut beraten, einen guten Notar und vielleicht noch weiteren Sachverstand hinzuzuziehen. Die Transaktionskosten hier sind vermutlich aus der Sicht der Beteiligten sinnvoll angewendet. So können wir allgemein festhalten, daß mit zunehmender Komplexität der wirtschaftlichen Austauschvorgänge auch nicht eine langfristige Senkung der Transaktionskosten zu erwarten ist. Im Gegenteil. In dem Maße, in dem als Folge des ungleichgewichtigen Wachstums (unbalanced growth) Extraktions-, Produktions- und Transportkosten säkular sinken, ist es nicht überraschend, daß vor allem die Transaktionskosten der Dienstleistung anteilsmäßig steigen werden. Insofern muß man erwarten, daß den Transaktionskosten mit zunehmender Komplexität der Volkswirtschaft eine zunehmende Bedeutung zuwächst (Wagners Gesetz). Daraus folgt aber weiterhin, daß die deshalb stets intensivere Suche nach einer Senkung dieser Kosten einen stets schnelleren Rechtswandel erfordern wird. Insofern wird auch dem evolutorischen Ansatz eine stets größere Bedeutung in der ökonomischen Rechtsanalyse zugemessen werden müssen.

\section{II.}

Die Abgrenzung des Rechtssystems läßt sich nicht unabhängig von den Regelungszusammenhängen vornehmen. Nur im einfachsten Fall (und daher selten) besteht das Rechtssystem aus der Legislative, der Jurisdiktion, der Exekutive und den Normadressaten. Das einfache viergliederige Schema läßt sich aber insofern gut als Ausgangspunkt heranziehen, als jedes Rechtssystem eben zumindest diese vier Elemente umfassen muß. Das System muß über eine Instanz verfügen, die die Normen verbindlich feststellt. Es muß eine Instanz geben, die die Normen verbindlich auf den Einzelfall anwendet. Es muß eine Instanz geben, die die Normeinhaltung überwacht. Und es muß schließlich Deutlichkeit darüber bestehen, für wen die Normen gelten sollen. Für den konkreten Fall der Analyse eines Regelungszusammenhangs sind alle vier Aspekte mindestens zu prüfen. Schmoller (1900, 1904; 1919) beschreibt im einzelnen, wie Markttransaktionen dort entstehen können, wo das Recht selbst gefestigt ist (das "alte Recht"). Hinzu kommen dann aber geografische Voraussetzungen, zum Beispiel die Lage eines Ortes an einem natürlichen Verkehrsweg, ökonomische Voraussetzungen (zum Beispiel die Stärke von Angebot und Nachfrage), politische Voraussetzungen (zum Beispiel der Landfrieden zur Verkehrssicherheit) und institutionelle Voraussetzungen (zum Beispiel die Verleihung eines Marktrechts, eines Stadtprivilegs, vielleicht sogar des Stapelrechtes oder des Münzrechtes. Beide Rechte sind wichtig im Hinblick auf die Transaktionskosten, erlaubt doch 
das Stapelrecht die Senkung der Informationskosten und das Münzrecht die Senkung der Kosten des Austausches. Schauen wir in diesem Fall auf die Rechtsquellen, so fällt sogleich auf, daß keine Legislative in Erscheinung tritt. Das Recht ist das überlieferte alte Recht, sowie es von den es anwendenden Spezialisten (das heißt hier den Kaufleuten, die dann den Rat der Stadt mitbilden) oft regelrecht handwerklich gelernt worden ist. Auch die Universitäten als öffentliche aber nicht staatliche Organe spielen ihre Rolle in der Tradition des Rechtes. Beide sind nicht Gesetzgeber, sondern das Recht ist evolutioniertes (gewachsenes) altes Recht das sich aus sich heraus erneuert. Es wird allerdings ergänzt durch Eingriffe von außen (Marktrecht, Stapelrecht, Münzrecht), sein Wandel wird also institutionell gefördert.

Findet der Austausch nicht öffentlich auf einem Markt statt, sondern innerhalb einer Organisation, so können Legislative, Jurisdiktion und Exekutive regelrecht zusammenfallen. Die Gilde, das Kloster, der Orden aber auch der Fronhof bilden selbstverständlich Rechtssysteme. Sombart (1904; 1916, 1927) arbeitet dies im Einzelnen heraus und weist insbesondere auf die Bedeutung der Minderheiten mit eigenen Rechts- und Glaubensvorstellungen hin. Der Ehrenkodex des Adels, der Glaube der verfolgten Minderheiten (Protestanten, Juden) sind auch Rechtsquellen, die für ein Rechtssystem dienen können und insofern den auf Vertrauen beruhenden Austausch, vor allem das Kreditgeschäft fördern und sichern.

In dem Maße, in dem neue wirtschaftliche Vorgänge entstehen, entstehen auch neue Rechtsformen und Rechtssysteme. Das Aufkommen einer professionellen auf klaren Prinzipien gegründeten Staatsverwaltung zu Beginn des 19. Jahrhunderts brachte es mit sich, daß ein Verwaltungsrecht entstand (Backhaus 1999a). Es hat gesetzte Rechtsquellen, aber beruht auch auf außerrechtlichen (vor allem ökonomischen) Prinzipien. Das Aufkommen des Sozialstaates gegen Ende des 19. Jahrhunderts brachte mit sich ein ausgeprägtes Sozialrecht. Die Institutionen des Arbeitsmarktes führten sehr bald auch zur Ausprägung eines Arbeitsrechts, das vom bürgerlichen Recht abgetrennt wurde. Dieses Beispiel zeigt deutlich, daß die institutionelle Ausgestaltung (hier des Arbeitsmarktes), die in den verschiedenen westlichen Ländern noch sehr unterschiedlich ist, auch $\mathrm{zu}$ unterschiedlichen Formen des Arbeitsrechts führen muß. Die Ausbildung gemeinsamer Prinzipien, die angesichts der zunehmenden internationalen Verflechtung der Arbeitsmärkte wichtig ist, ist selbst eine bedeutende Aufgabe auch für die ökonomische Rechtsanalyse.

\section{III.}

Der Systemwandel im Recht kann einerseits endogen erfolgen; hierauf richten vor allem Rechtsökonomen in der Chicago-Tradition (Posner 1971; 1977, 1986) ihr Augenmerk. Die Vorstellung unter dem Stichwort die Effizienz des Common Law besteht darin, daß sich das angloamerikanische Common Law in einem evolutorischen Prozeß stets verfeinert, denn die schlechteren Lösungen werden von den Parteien abgelehnt und selbst passive Richter so dazu 
bewogen, mit Berufungsklagen konfrontiert auf offensichtlich bessere Vorschläge der Parteien einzugehen (Backhaus 1998a).

Ebenfalls zum endogenen Systemwandel des Rechts gehört die Kodifikation (Backhaus 1999b, The German Civil Code). Ob eine Kodifikation wünschenswert ist, oder ob die Jurisdiktion aus sich heraus das Recht erneuern kann, ist eine Frage, die in der Rechtsgeschichte immer wieder zu heftigen Kontroversen Anlaß gegeben hat. Man denke etwa an den erbitterten Streit zwischen Thibaut (1772-1840) und Von Savigny (1779-1861), die Auseinandersetzungen und das deutsche bürgerliche Gesetzbuch von 1900 und die neuerlichen Überlegungen hinsichtlich eines gemeinsamen europäischen Bürgerlichen- und Handelsgesetzbuches. Beide Möglichkeiten, die kontinuierlichen Fortführung der Rechtsprechung und die grundlegende Erneuerung durch Kodifikation können evolutionär erfolgen. Nur sieht man einmal die Legislative als Teil des evolutionären Systems, im anderen Falle als einen Außeneinfluß. Wie wir das Rechtssystem definieren ist hier von großer Bedeutung, denn das Subsidiaritätsprinzip verlangt ja im europäischen Recht, daß das kleinere funktionsfähige Rechtssystem auch die Kompetenz erhält. Wenn also dargestellt werden kann, daß das kleinere, untere oder in jeden Fall bürgernähere Rechtssystem tatsächlich funktionsfähig ist, dann fällt ihm dem Subsidiaritätsprinzip zu Folge auch den Kompetenz zu (Backhaus 1998b).

In jedem Fall beruht eine Kodifikation auf der sorgfältigen Rechtstatsachenforschung, um jene empirisch erprobten Rechtslösungen zu kodifizieren, die sich nachweislich bewährt haben und zu denen es auch eine ausreichende Rechtsprechungspraxis gibt. Die Betonung der Bedeutung einer solchen Rechtstatsachenerhebung und ihre Verwendung im Kodifikationsprozeß gab auch Anlaß zur Revision des ersten Entwurfes des deutschen bürgerlichen Gesetzbuches auf Grund der Arbeiten Otto von Gierkes (Backhaus 1999c).

\section{Zusammenfassung und Ausblick}

Der evolutorische Ansatz zur ökonomischen Rechtsanalyse wird sich in den folgenden Jahren mehrfach zu bewähren haben. Die Europäische Kommission versucht ganze Märkte neu zu strukturieren, zu denken ist an den Buchhandel, den Automobilhandel, den Handel mit anderen vor allem durch Markenzeichen beschriebenen Gütern (zum Beispiel Parfums) und den Handel mit forschungsintensiven Produkten, die gleichzeitig sehr stark im sozialpolitischen Interesse stehen, wie zum Beispiel Pharmazeutika. Die Marktstrukturanalysen, die die Europäische Kommission zu Grunde legt, sind noch sehr rudimentär. Das Problem der Transaktionskosten spielt erst ganz am Rande eine Rolle und wird zunehmend in dem Mittelpunkt rücken müssen, da die Markteingriffe der Kommission, wenn sie nicht erfolgreich sind, zu einer Verschiebung der Märkte außerhalb des europäischen Vertragsgebietes führen werden.

Auch Verfassungsstrukturen, in denen also das gesamte Rechtssystem zur Disposition steht, sind im Rahmen des europäischen Einigungsprozesses ständigen Erprobungen ausgesetzt. 
Dies sieht man insbesondere in der Haltung des deutschen Bundesverfassungsgerichtes, da es anders als die Gerichte anderer Mitgliedstaaten allein zum Hüter der Verfassung aufgerufen ist, eine Aufgabe, die es etwa im Vergleich mit dem französischen Conseil d'État weit auffasst. In dem Maße, in dem der europäische Einigungsprozeß am Verfassungsverständnis einzelner oder mehrerer Mitgliedstaaten vorbeigeht, wird es zu erheblichen Verwerfungen führen, die man mit Hilfe eines evolutorischen Ansatzes sehr gut analysieren kann.

Schließlich bleibt noch die Frage des Rechtswandels auf Grund großer legislativer Projekte. Bislang hat die Europäische Union, von der Einführung der Mehrwertsteuer allenthalben abgesehen, noch nicht viel in Angriff genommen. Der Versuch eines gemeinsamen europäischen Gesellschaftsrechts ist über den Stand der 70. Jahre bislang nicht wesentlich hinausgelangt. Da es in der Natur der Sache liegt, daß die Europäische Gemeinschaft in Rat, Kommission oder Parlament diese Projekte vorantreiben wird, ergibt sich ein weites Feld der Anwendung evolutorischer Ansätze zur ökonomischen Rechtsanalyse.

\section{Literaturverzeichnis}

Backhaus, Jürgen G. (1998a), "Efficient Statute Law", in: Peter Newman (Hrsg.), The New Palgrave Dictionary of Economics and the Law, London: MacMillan, II, S. 24-28

Backhaus, Jürgen G. (1998b), "Das Subsidiaritätsprinzip als Baustein einer entstehenden europäischen Verfassung", in: Dieter Cassel (Hrsg.), Europäische Integration als ordnungspolitische Gestaltungsaufgabe; Probleme der Vertiefung und Erweiterung der Europäischen Union, Berlin: Duncker \& Humblot, 1998, S. 79-94

Backhaus, Jürgen G. (1999a), (Hrsg.) Elgar Companion to Law and Economics, Cheltenham: Edward Elgar, 1999, S. 561

Backhaus, Jürgen G. (1999b), "The German Civil Code of 1896: An Economic Interpretation", European Journal of Law and Economis, vol. 7, nr. 1, S. 5-14

Backhaus, Jürgen G. (1999c), "Otto von Gierke", in: Jürgen G. Backhaus (Hrsg.) Elgar Companion to Law and Economics, Cheltenham: Edward Elgar, S. 313-315

Coase, Ronald H. (1937), "The Nature of the Firm", Economica 4, S. 387-405

Posner, Richard A. (1971; 1977, 1986), "Economic Analysis of Law", Boston: Little Brown

Schmoller, Gustav (1900, 1904; 1919), "Grundriß der allgemeinen Volkswirtschaftslehre", München/Leipzig: Duncker \& Humblot

Sombart, Werner (1904; 1916, 1927), "Der moderne Kapitalismus", München/Leipzig: Duncker \& Humblot

Witt, Ulrich (1994), "What Evolutionary Economics is all about", in Ulrich Witt (Hrsg.) Evolutionary Economics, Cheltenham: Edward Elgar, S. 83 